\title{
GSC 1.2 - A SUBPLATE REDUCTION OF THE GSC
}

\author{
B. BUCCIARELLI ${ }^{1}$, J.B. DOGGETT ${ }^{1}$, C.R. STURCH ${ }^{1,2}$, B.M. LASKER ${ }^{1}$, B.J. \\ MCLEAN $^{1}$, M.G. LATTANZI ${ }^{1,3}$ and L.G. TAFF ${ }^{1}$ \\ ${ }^{1}$ Space Telescope Science Institute, Baltimore, MD 21218, U.S.A. \\ ${ }^{2}$ Astronomy Programs, Computer Sciences Corporation \\ ${ }^{3}$ Astrophys. Dept. SSD, ESA, \\ on leave from Osservatorio Astronomico di Torino
}

\section{Introduction}

The original version of the HST Guide Star Catalog (GSC 1.0, Lasker et al. 1990, and following papers) contains systematic errors which typically begin 1.5 from the plate center and increase with radius (Taff et al. 1990a). In a residual plot against CAMC stars this shows as high correlations between neighboring residuals. This is due to the inability of a global polynomial to map the metric of a Schmidt plate. A new reduction of the GSC measures (GSC 1.2) based on the subplate method (Taff 1989) is partially complete. We report on it for the northern data from the Palomar Quick V plates, with 20 minute exposures, nearly all taken in the 1982-1984 interval.

\section{The Subplate Method}

The GSC 1.2 reduction is based on the subplate method, which has been proven to remove the systematic residuals affecting polynomial solutions (Taff et al. 1990b). The deformations in Schmidt plate geometry are local in nature. Accordingly, the subplate method treats the Schmidt plate as many small, overlapping sub-areas. They must be small enough to be described by a linear metric, but large enough for good reference star statistics. The reference catalogue used for the new reduction is the PPM (Roeser \& Bastian 1991), with a nominal northern hemisphere positional error of 0.38 at 1990 . A typical subplate size is $1^{\circ}$, so the adopted $1 / 3$ overlapping yields, on the average, 9 independent reference stars per subplate.

\section{Astrometric Errors}

The search for systematic errors which depend on plate position is conducted by constructing a super-plate, whereon the CAMC-GSC vector residuals are placed, binned and averaged. The resulting map smooths the random noise, thus unveiling any plate-based signature. Maps, representing systematic departures from the plate model over a quarter of the plate, are shown in 
Fig. 1. Each grid point is summed over an area of $\left(10^{\prime}\right)^{2}$. There is a dramatic improvement from the GSC 1.0 map to the GSC 1.2. The absolute positional errors versus distance are given in Fig. 2. The GSC 1.2 curve is much flatter up to $\sim 150^{\prime}$, then deteriorates further out.

The relative errors between pairs of stars as a function of their separation are illustrated in Fig. 3. The subplate solution is more accurate than the GSC 1.0 solution, but limitations on the density of reference stars make it incapable of eliminating systematics below the half-degree level. Finally, present analysis leads to an expectation of $\sim 0.45$ average positional error for GSC 1.2.
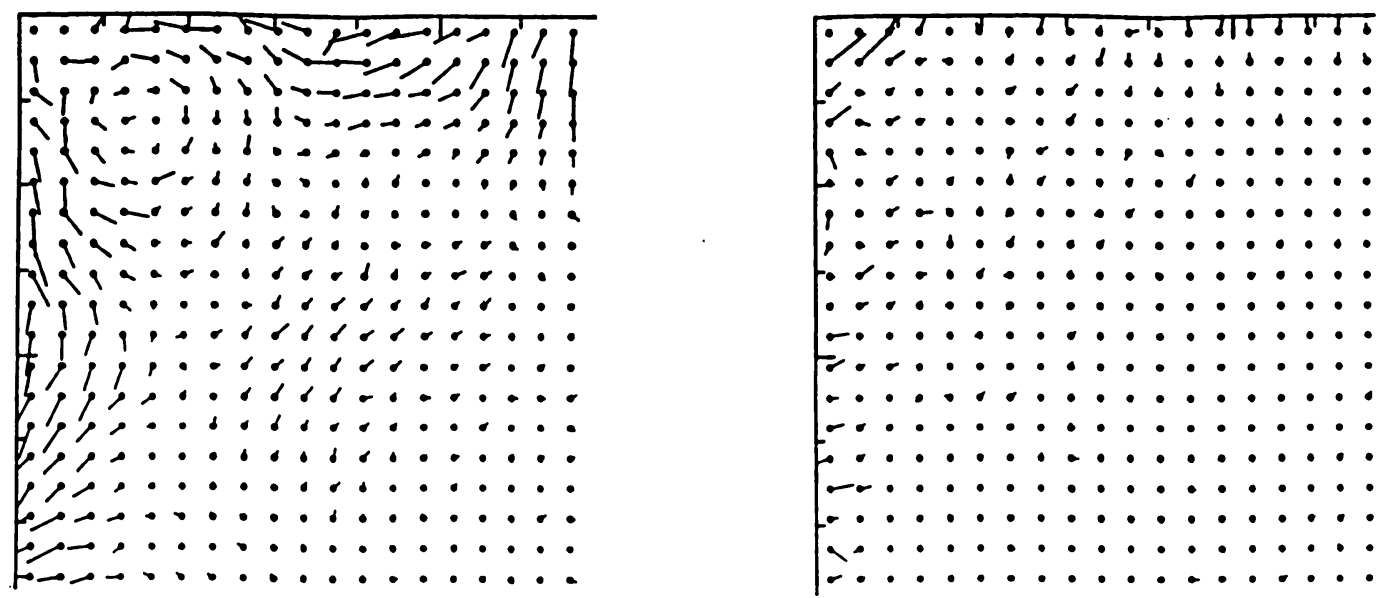

Figure 1. (a) Systematic departures from the plate model GSC 1.0; (b) the same for GSC 1.2.

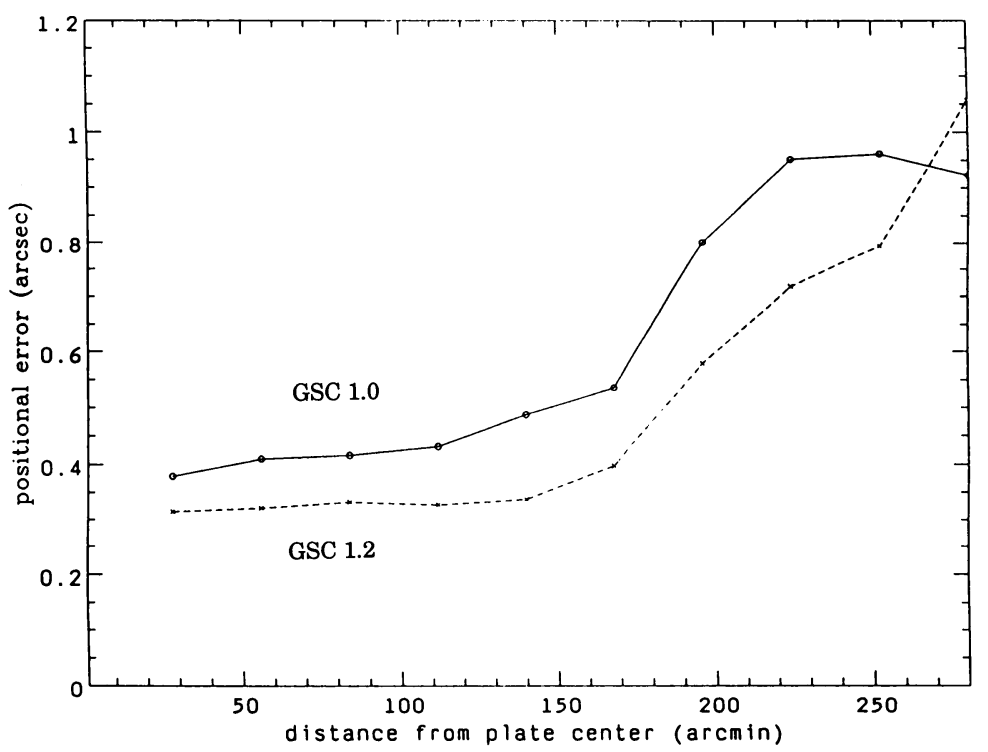

Figure 2. Average positional errors vs. distance from plate center. 


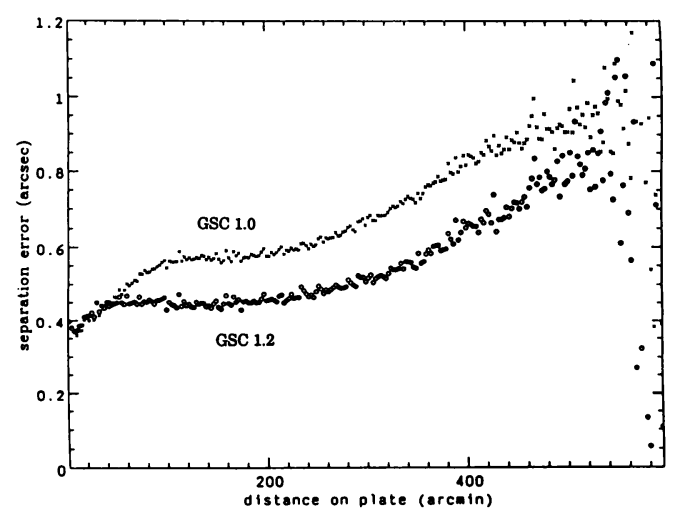

Figure 3. Relative errors for pairs of stars.

\section{References}

CAMC Consortium 1991. Carlsberg Meridian Catalog, La Palma (Copenhagen Univ. Obs., Copenhagen), Number 5.

Lasker, B.M., Sturch, C.R. McLean, B.J., Russell, J.L., Jenkner, H. and Shara, M.M., 1990. Astron. J., 99, 2019.

Roeser, S. and Bastian, U., 1991. PPM Star Catalogue Astronomisches Rechen Institut, 2 vols. Taff, L.G., 1989. Astron. J., 98, 1912.

Taff, L.G., Lattanzi, M.G., Bucciarelli, B., Gilmozzi, R., McLean, B.J., Jenkner, H., Laidler, V.G., Lasker, B.M., Shara, M.M. and Sturch, C.R., 1990a. Astrophys. J. Letter, 353, L45.

Taff, L.G., Lattanzi, M.G. and Bucciarelli, B., 1990b. Astrophys. J., 358, 359. 